\title{
Molecular Dynamics Simulations of Prion Peptides: Effect of Glycosylation
}

\author{
Youngjin Choi, Hyunmyung Kim, ${ }^{\dagger, \neq}$ Suntae Hwang, ${ }^{\S}$ Karpjoo Jeong, ${ }^{\dagger,+, *}$ and Seunho Jung ${ }^{\ddagger, \#, *}$ \\ BioChip Research Center, Hoseo University, Asan 336-795, Korea \\ ${ }^{\dagger}$ Department of Advanced Technology Fusion, Konkuk University, Seoul 143-701, Korea. *E-mail: jeongk@konkuk.ac.kr \\ ${ }^{\star}$ Research Institute for Ubiquitous Information Technologies and Applications (UBITA), \\ Konkuk University, Seoul 143-701, Korea \\ ${ }^{\S}$ Department of Computer Science, Kookmin University, Seoul 136-702, Korea \\ ${ }^{\#}$ Department of Bioscience and Biotechnology, Center for Biotechnology Renovation in Ubita, Konkuk University, \\ Seoul 143-701, Korea. *E-mail: shjung@konkuk.ac.kr \\ Received November 16, 2010, Accepted December 14, 2010
}

Key Words: Glycosylation, Single amino acid substitution, Amyloid formation

\begin{abstract}
Glycoproteins are classes of naturally occurring bioconjugates in which side chains of the protein moieties are modified either by $N$-linked and $O$-linked oligosaccharides. Glycosylation can affect biophysical properties such as solubility, thermal stability, aggregation, folding, and structural dynamics. ${ }^{1-3} \mathrm{Al}-$ though much is known about their composition or biosynthesis of oligosaccharides attached to proteins, it is not entirely clear how glycosylation contributes to the whole structure and function of glycoprotein. ${ }^{1,3}$
\end{abstract}

It is reported that $O$-glycosylation usually changes the conformational properties of protein backbone, and therefore also affects the kinetics of amyloidosis process. Amyloid is defined as a certain aggregated condition of proteins with showing deposits in various tissues. ${ }^{4}$ Aggregation process is generally triggered by abnormal $\beta$-sheet formation, allowing tight molecular interaction in a protein level. ${ }^{4}$ This feature is indispensable part for the occurrence of the infectious prion protein. The rate of fibrillogenesis of $\alpha$-helical hairpin model peptide is largely affected by chemical modifications including glycosylation. ${ }^{2}$ Single $O$-linked sugar affected structural transition in hamster prion peptide from random coil to $\beta$-structure. ${ }^{5,6} O$-glycosylation with an $\alpha$-linked sugar ( $\alpha$-GlcNAc or $\alpha$-GalNAc) on Ser 135 showed a dramatic inhibitory effect on amyloid formation in the prion peptide. However, R136G mutation highly increased amyloid formation of this mutant peptide. ${ }^{6}$

Prion diseases are a group of neurodegenerative disorders which involve an improper folding of the prion protein (PrP) characterized by problematic conversion into the aggregated form. The misfolded prion protein $\left(\mathrm{PrP}^{\mathrm{Sc}}\right)$ is regarded as a template to cause this abnormality into new normal $\left(\operatorname{PrP}^{\mathrm{C}}\right)$ state and is the proposed mechanism behind the infectivity of prions. ${ }^{7}$ The distinctive features of this aggregated form are decreased $\alpha$-helical portion and increased $\beta$-sheet content. ${ }^{8}$ These changes in secondary structure of the protein were also linked by aggregation process during the amyloidosis. The structural changes found in the human prion sequence 90-144 were directly relevant to the transmission of prion diseases. ${ }^{9,10}$ Furthermore, the peptide 90 - 145 caused $\beta$-structures when dissolved in aqueous solution at physiological salt concentration. ${ }^{11}$ The solution structure of recombinant syrian hamster prion protein $(90$ - 231) was determined $^{12}$ with missing residues $(90-124)$. The $\mathrm{N}$-terminus
(90 - 112) is largely unstructured and the segment residues 113 - 125 forming hydrophobic cluster is not well defined.

Molecular dynamics (MD) simulations are prevalent technique to gain a profound grasp on the biomolecular structure or conformational transition. We now report comparative MD studies of prion peptide 108 - 144, R136G mutant, and glycosylated forms to derive influence of glycosylation upon conformational changes of prion peptide (Fig. 1). It has been reported that just one $O$-linked sugar can affect the properties of conformational transition and amyloid formation in the prion peptide. ${ }^{5,6}$ Because there was no experimentally solved structure of 108 - 122 sequence in hamster prion, we first executed the conformational sampling of the peptides using computational method. The structure of peptides was initially on the random coil, then transformed into the amyloid structure. ${ }^{13}$ Combination of replica-exchange simulations with simulated tempering (SAREMD) was applied for the conformational sampling of the peptides. Using the conformation generated by SA-REMD as initial conformation, conventional molecular simulations were used to trace the conformational transition of the prion peptides.

SA-REMD simulations of prion peptides in implicit solvent were performed to obtain the folded structure. There are many references on the REMD method to study amyloid peptide using molecular simulation. ${ }^{14-16}$ Replica exchange process is known to be effective in sampling more low-energy decoys compared to SA-MD simulation. ${ }^{17}$ In the case of SA-MD simulations, native-like structures were sampled at some stage but these states were frequently lost during the simulations. In the present study, SA-REMD simulations were started at $710 \mathrm{~K}$ with a final target temperature of $310 \mathrm{~K}$. Fig. 2 shows the final and the secondary structure of peptides sampled during the SA-REMD simulation for the replica run at the lowest temperature. All

$\begin{array}{ll} & \\ \text { Peptide G } & \text { Ac-NMK HMAGAAAAGA VVGGLGGYML GSAMSRPMMH FGND-NH } \\ & \\ \text { Peptide } S & \text { Ac-NMK HMAGAAAAGA VVGGLGGYML GSAMSGPMMH FGND-NH } \\ & \\ \text { Peptide C } & \text { Ac-NMK HMAGAAAAGA VVGGLGGYML GSAMSRPMMH FGND-NH }\end{array}$

Figure 1. Amino-acid sequences of native (108 - 144; peptide C), glycosylated (peptide $\mathrm{G}$ ) and substituted (peptide $\mathrm{S}$ ) prion peptide. The site of glycosylation and substitution are underlined. 
(A)
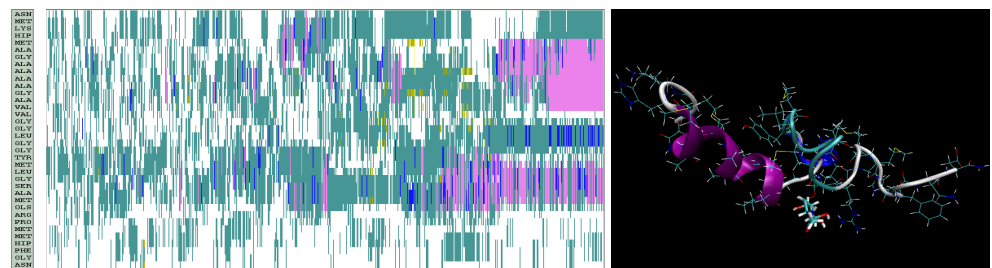

(B)
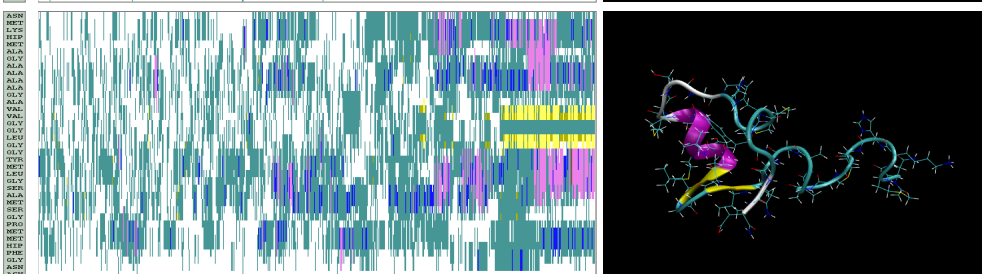

(C)
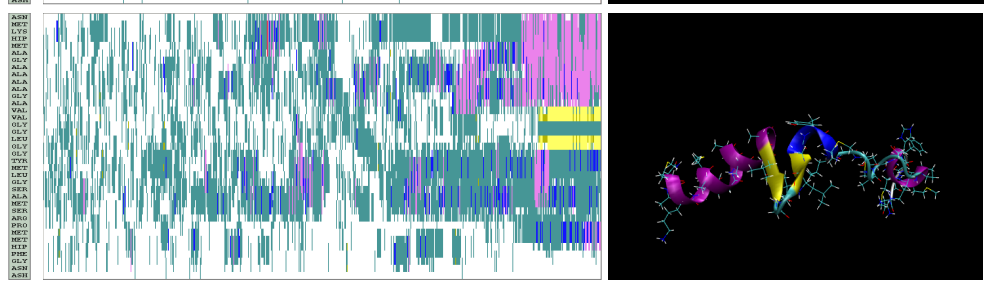

Figure 2. Secondary structure changes and final conformations of $O$-glycosylated (A), R136G (B), and Native (C) peptides during the simulated annealing coupled REMD simulations (with 4 replicas) for the replica run at the lowest temperature $(710 \mathrm{~K} \rightarrow 310 \mathrm{~K})$. Dashed gray lines indicate the $O$-glycosylated serine residue (A) and mutated (Arg $\rightarrow$ Gly) residue (B) respectively.

(A)

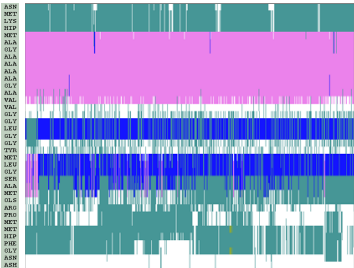

(B)
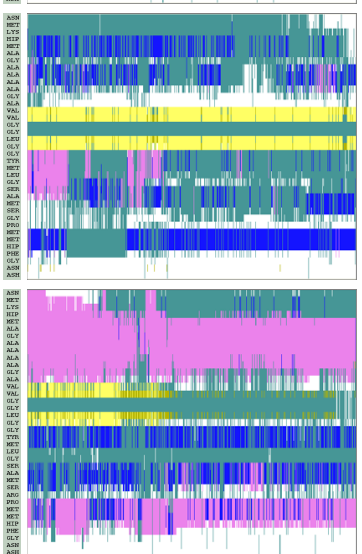
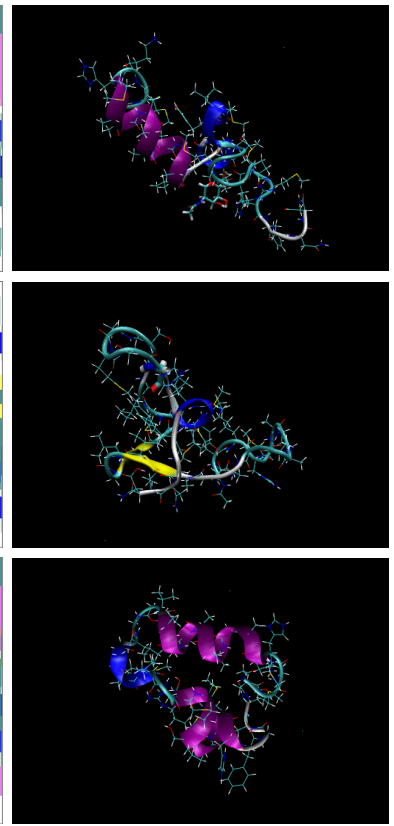
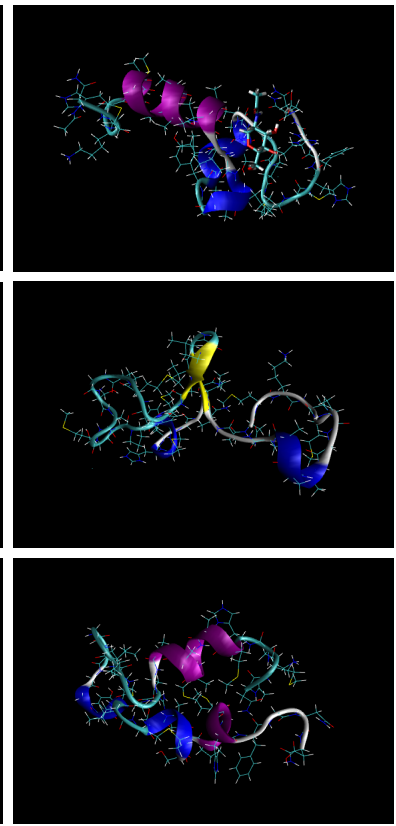

Figure 3. Molecular dynamics simulations at $310 \mathrm{~K}$ in aqueous environment. The initial conformations of peptides are obtained from a simulated annealing coupled replica exchange molecular dynamics (REMD). Secondary structure changes during $15 \mathrm{~ns}$ MD simulations and representative conformations of peptides (left) at $7 \mathrm{~ns}$ (middle) and $15 \mathrm{~ns}$ (right) are shown, respectively.

three peptide exhibits the $\alpha$-helical and turn structures during the final $0.25 \mathrm{~ns}$ at the lowest temperature of MD simulations. However, the $\beta$-sheet region is observed at the late phase of simulations only for the native or R136G mutant peptide. The $O$-glycosylated form shows no clear $\beta$-sheet structure during the whole stage of SA-REMD simulations.

The conformational transition of prion peptides under experimental conditions was investigated by conventional MD simulations using explicit solvent at $310 \mathrm{~K}$. The final structures of prion peptides from SA-REMD were used as an initial conformation for each MD run. During the 15 ns of MD time scale, glycosylated peptide showed neither $\beta$-sheet transition nor large structural changes, which is comparable to the results of SA-REMD. (Fig. 3) The Ala-120 and Val-121 acted as $\beta$-sheet formers at both native and R136G peptide. R136G peptide maintained original $\beta$-sheet structure during the whole MD simulations with gradual decreasing in the $\alpha$-helix content.

Although the exact conformation of the seed structure has 
(A)

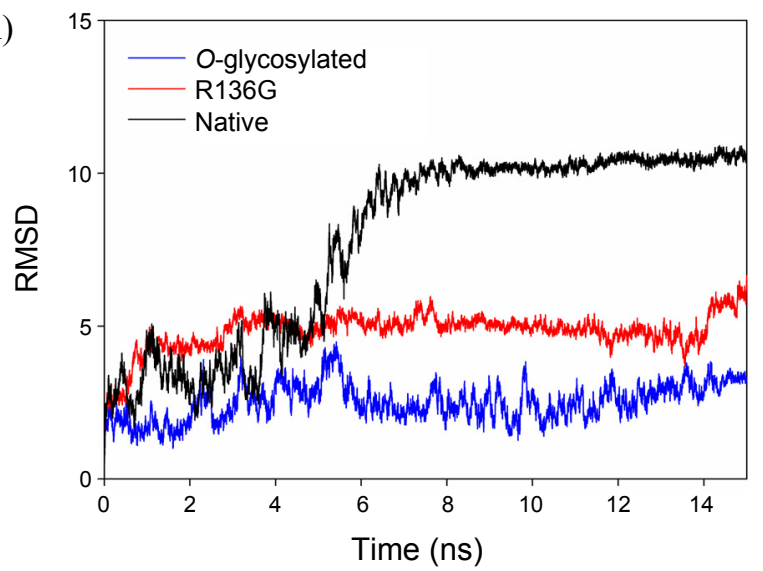

(B)

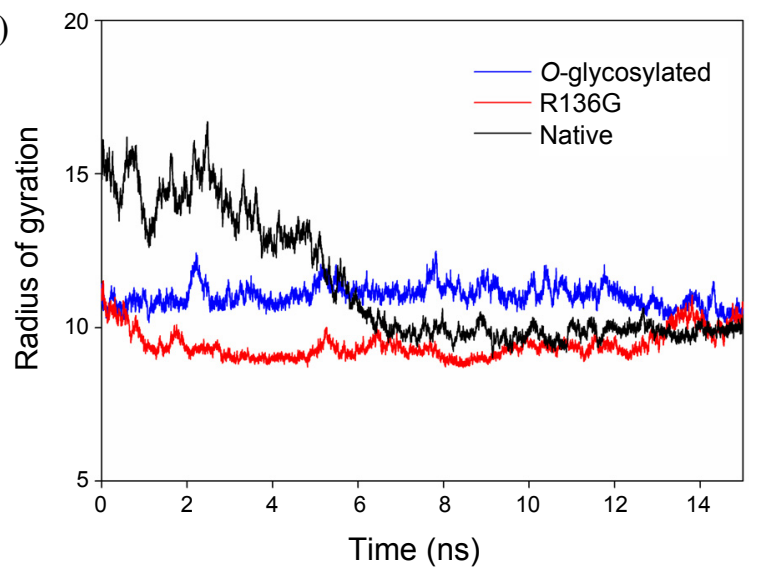

Figure 4. Time evolution for the RMSD of $\mathrm{C} \alpha$ atoms (A) and the radius of gyration (B) of each peptide during the molecular dynamics simulations. The RMSD of each peptide was compared to the initial structure derived from the simulated annealing coupled REMD simulations. not been known, but amyloid fibrils share the common $\beta$-sheet structure. Even though $\beta$-sheet accounts for only minor portion in the peptide, it can be a 'hot spot' that provides driving force to the aggregation process of amyloid peptides. ${ }^{18}$ Therefore, the conformational transition into $\beta$-sheet structure might be an initial step to build fibrillar structure in the prion peptide. Highly retarded amyloid formations of $O$-glycosylated prions are might be due to this reduced $\beta$-sheet formation.

The effect of site modification on the whole conformation of the peptide bond adjacent to the modified residue was investigated in terms of molecular flexibility. As secondary structures of each peptide form changed during MD simulations, conformational conversion was initiated. The RMSD values were checked to confirm less flexible structure of glycosylated prion peptide (Fig. 4A). It appears that sudden increase in C $\alpha$ RMSD of native peptide observed from 5.0 to $8.0 \mathrm{~ns}$ corresponds with the deterioration of the secondary structure. However, the glycosylated form showed the lowest RMSD changes compared to both native and R136G mutant. That means the glycosylation forced to maintain rigid prion conformation during MD simulations. The flexibility was not related with molecular size effect which confirmed by radius of gyration $\left(R_{\mathrm{G}}\right)$. The $R_{\mathrm{G}}$ values were converged to the level of $10 \AA^{2}$ among three different peptide forms with showing slightly higher values in the glycosylated form caused by additional sugar moiety (Fig. 4B).

Local dihedral flexibility changes induced by modification were also analyzed using by the popular density map of selected dihedral angles of each peptide (Fig. 5). Protein conformation is mainly affected by both backbone and side-chain flexibility. It is reasonable to assume the local flexibility of each prion using dihedral angle distribution form the popular density map. The glycosylation showed only slight increase in dihedral angle distribution at Ser135 (OLS, $O$-Linked Sugar) whereas the R135G

(A)
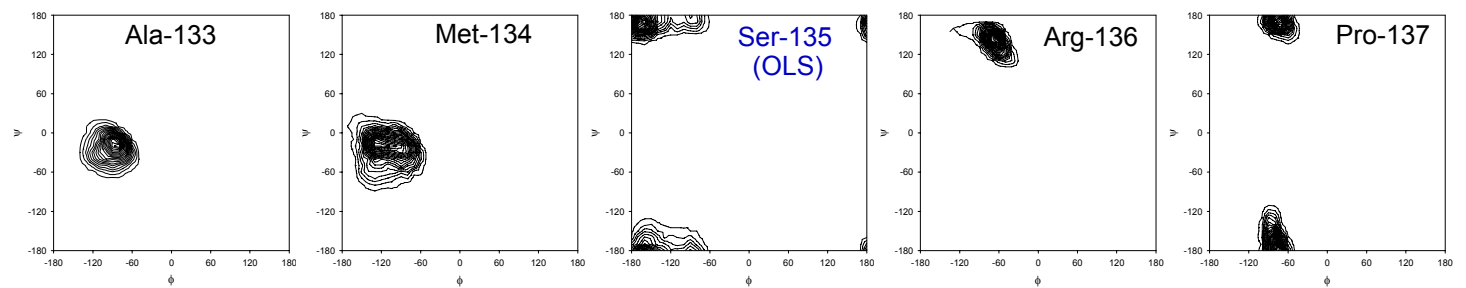

(B)
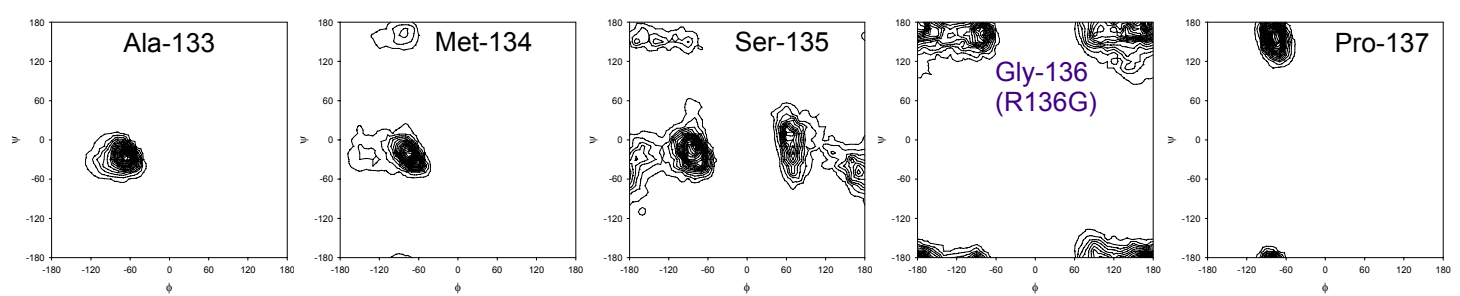

(C)
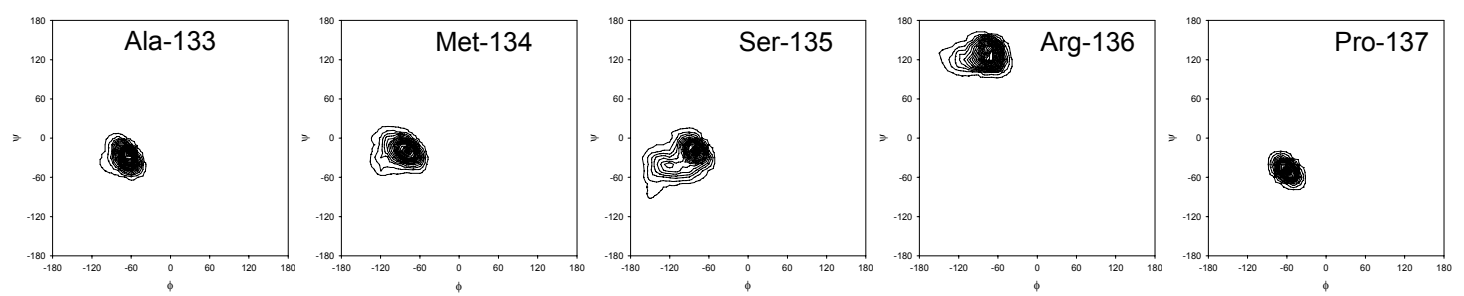

Figure 5. Popular density map of selected dihedral angles of $O$-glycosylated (A), R136G (B), and Native (C) peptides during the 15 ns molecular dynamics simulations. 
mutation caused considerable rising in the angle distribution at Met134, Ser135, and Gly135. These results mean that glycosylation only give a minor perturbation effect on the local structure around the glycosylation site. Therefore the glycosylation enhances molecular interaction within the prion structure using additional sugar residue compared to the native prion peptide. In this study, we demonstrated $\alpha$-GlcNAc moiety could affect the peptide conformation by interacting with not only adjacent residue (Arg136) but also distal residues (Ala120, Val121) located in $\alpha$-helix structure. This interaction can help to maintain original structure without sudden conformational changes.

\section{Computational Methods}

Molecular Models and SA-REMD Simulations. The sander module of the AMBER 10 package ${ }^{19}$ was used in combination with the parm03 and GLYCAM_06 force field. ${ }^{20}$ An advanced Generalized Born implicit solvent model was applied to SAREMD simulations. ${ }^{21}$ Starting structures were generated using xleap module of the AMBER 10 package as a fully extended form. The glycosylated for was built with $\alpha$-GlcNac on the Ser135 residue of the prion peptide. The annealing was performed in 20 steps with a starting temperature of $710 \mathrm{~K}$ and a final target temperature of $310 \mathrm{~K}$; all the temperatures in Kelvin: $310,325,240,355,370,385,400,420,440,460,480,500,520$, $540,565,590,615,640,670,710$. Replica exchanges between 4 replica simulations at 4 neighboring temperatures were performed. Exchanges were attempted every 1 ps according to a standard Metropolis criterion for the potential energy of the system. SA-REMD were started at the 4 highest neighboring temperature and annealing was performed by reducing simulation temperatures by one temperature step until the lowest temperature was reached $310 \mathrm{~K}$ in one of the replica.

MD Simulations in an Aqueous Environment. The initial structures were taken from the final structure of SA-REMD simulations. Peptides were solvated in a cubic box filled with TIP3P water ${ }^{22}$ molecules ( 4200). A 2 fs time step was used in all the simulations, and long-range electrostatic interactions were treated with the particle mesh Ewald (PME) procedure ${ }^{23}$ with a 12 Å nonbonded cutoff. Bond lengths involving hydrogen atoms were constrained using the $\mathrm{SHAKE}^{24}$ algorithm. Systems were energy-minimized prior to the production run. The solvent molecules were first relaxed, while all atoms of peptides were restrained with forces of $500 \mathrm{kcal} \mathrm{mol}^{-1} \AA^{-2}$. The systems were then continually relaxed. Finally, all restraints were lifted and the whole system was relaxed with 2000 cycles of steepest descent followed by 3000 cycles of conjugate gradient minimization. After relaxation, 200 ps MD simulations were carried out at constant volume, with $10 \mathrm{kcal} \mathrm{mol}^{-1} \AA^{-2}$ restraints on the solute. Then, 15 ns NPT MD simulations were carried out on systems at constant pressure (1 atm). All simulations were performed at $310 \mathrm{~K}$ except the equilibrium MD run. The ptraj module of AMBER was used to analyze the results. The RMSD values were calculated only for $\mathrm{C}_{\alpha}$ of peptides. The hydrogen bonds were defined as hydrogen acceptor-donor atom distances of less than $3.5 \AA$ and acceptor-H-donor angles of more than $120^{\circ}$. Molecular visualization and secondary structure of the peptides were produced using the program VMD and the STRIDE module within the VMD software.

Acknowledgments. This work was supported by Priority Research Centers Program through the National Research Foundation of Korea (NRF) funded by the Ministry of Education, Science and Technology (2009-0093824).

\section{References}

1. Wang, C.; Eufemi, M.; Turano, C.; Giartosio, A. Biochemistry 1996, 35, 7299.

2. Liang, F.; Chen, R.; Lin, C.; Huang, K.; Chan, S. Biochem. Biophys. Res. Commun. 2006, 342, 482.

3. Spiriti, J.; Bogani, F.; van der Vaart, A.; Ghirlanda, G. Biophys. Chem. 2008, 134, 157.

4. Westermark, G. T.; Westermark, P. Trend. Mol. Med. 2010, 16, 501.

5. Chen, P.; Lin, C.; Chang, Y.; Lin, S.; Chan, S. Proc. Natl. Acad. Sci. USA 2002, 99, 12633.

6. Ho, C.; Lee, L.; Huang, K.; Lin, C.; Ku, M.; Yang, C.; Chan, S.; Hsu, R.; Chen, R. Proteins 2009, 76, 213.

7. Prusiner, S. B. Science 1982, 216, 136.

8. Caughey, B. W.; Dong, A.; Bhat, K. S.; Ernst, D.; Hayes, S. F.; Caughey, W. S. Biochemistry 1991, 30, 7672.

9. Kaneko, K.; Ball, H. L.; Wille, H.; Zhang, H.; Groth, D.; Torchia, M.; Tremblay, P.; Safar, J.; Prusiner, S. B.; DeArmond, S. J.; Baldwin, M. A.; Cohen, F. E. J. Mol. Biol. 2000, 295, 997.

10. Kaneko, K.; Peretz, D.; Pan, K. M.; Blochberger, T. C.; Wille, H.; Gabizon, R.; Griffith, O. H.; Cohen, F. E.; Baldwin, M. A.; Prusiner, S. B. Proc. Natl. Acad. Sci. USA 1995, 92, 11160.

11. Zhang, H.; Kaneko, K.; Nguyen, J. T.; Livshits, T. L.; Baldwin, M. A.; Cohen, F. E.; James, T. L.; Prusiner, S. B. J. Mol. Biol. 1995, 250, 514.

12. James, T. L.; Liu, H.; Ulyanov, N. B.; Farr-Jones, S.; Zhang, H.; Donne, D. G.; Kaneko, K.; Groth, D.; Mehlhorn, I.; Prusiner, S. B.; Cohen, F. E. Proc. Natl. Acad. Sci. USA 1997, 94, 10086.

13. Lipfert, J.; Franklin, J.; Wu, F.; Doniach, S. J. Mol. Biol. 2005, 349,648

14. Jang, S.; Shin, S. J. Phys. Chem. B Lett. 2006, 110, 1955.

15. Simone, A. D.; Esposito, L.; Pedone, C.; Vitagliano, L. Biophys. J. 2008, 95, 1965.

16. Zhuang, W.; Sgourakis, N. G.; Li, Z.; Garcia, A. E.; Mukamel, S. Proc. Natl. Acad. Sci. USA 2010, 107, 15687.

17. Kannan, S.; Zacharias, M. J. Struct. Biol. 2009, 166, 288.

18. Sánchez, N.; Pallarés, I.; Avilés, F.; Vendrell, J.; Ventura, S. BMC Struct Biol. 2005, 5, 18.

19. Case, D. A.; Cheatham, T. E., III.; Darden, T.; Gohlke, H.; Luo, R.; Merz, K. M., Jr.; Onufriev, A.; Simmerling, C.; Wang, B.; Woods, R. J. Computat. Chem. 2005, 26, 1668.

20. Kirschner, K. N.; Yongye, A. B.; Tschampel, S. M.; Daniels, C. R.; Foley, B. L.; Woods, R. J. J. Comput. Chem. 2008, 29, 622.

21. Pitera, J. D.; Swope, W. Proc. Natl. Acad. Sci. USA 2003, 100, 7587.

22. Jorgensen, W. L. J. Chem. Phys. 1982, 77, 4156.

23. Darden, T.; York, D.; Pedersen, L. J. Chem. Phys. 1993, 98, 10089.

24. Ryckaert, J. P.; Ciccotti, G.; Berendsen, H. J. C. J. Comput. Phys. 1977, 23, 327 . 